\title{
Manipulating DC Currents with Bilayer Bulk Natural Materials
}

\author{
Tiancheng Han, Huapeng Ye, Yu Luo, Swee Ping Yeo, Jinghua Teng, \\ Shuang Zhang, and Cheng-Wei Qiu**
}

The principle of transformation optics has been applied to various wave phenomena (e.g., optics, electromagnetics, acoustics, and thermodynamics). Recently, metamaterial devices manipulating DC currents have received increasing attention, which usually adopted the analogue of transformation optics using complicated resistor networks to mimic inhomogeneous and anisotropic conductivities. We propose a distinct and general principle of manipulating DC currents by directly solving electric conduction equations, which only needs to utilize two layers of bulk natural materials. We experimentally demonstrate DC bilayer cloaks and fan-shaped concentrators, derived from the generalized account for cloaking sensors. The proposed schemes have been validated as exact devices and this opens a facile way towards complete spatial control of DC currents. The proposed schemes may have vast potential in various applications not only in DC but also in other fields of manipulating magnetic fields, thermal heat, elastic mechanics, and matter waves.

Controlling electromagnetic (EM) fields so as to render an object invisible has been a long-standing dream for many researchers over the decades. ${ }^{[1,2]}$ On the basis of the invariance of Maxwell's equations where equivalence is established between metric transformations and changes in material parameters, transformation optics ${ }^{[3]}$ and conformal mapping ${ }^{[4]}$ have been developed to manipulate EM wave propagation in a practically arbitrary manner. Besides making objects invisible, ${ }^{[1-4]}$ many other novel devices are rapidly emerging, with a representative one being a concentrator ${ }^{[5,6]}$ that can enhance the energy density of incident waves in a given area. In addition

Dr. T. Han, H. Ye, Prof. S. P. Yeo, Prof. C.-W. Qiu Department of Electrical and Computer Engineering National University of Singapore

117583, Republic of Singapore

E-mail: chengwei.qiu@nus.edu.sg

Dr. Y. Luo

Department of Physics

Blackett Laboratory

Imperial College London

London, SW7 2AZ, UK

Prof. J. Teng

Institute of Materials Research and Engineering

Agency for Science, Technology and Research, 3 Research Link

Singapore 117602, Singapore

Prof. S. Zhang

School of Physics and Astronomy

University of Birmingham

Birmingham, B15 2TT, UK

DOI: 10.1002/adma.201305586 to manipulation of EM waves, ${ }^{[1-6]}$ the theoretical tool of coordinate transformation has been extended to other areas of physics (such as acoustic waves, ${ }^{[7]}$ matter waves, ${ }^{[8]}$ and elastic waves ${ }^{[9]}$ ).

Recently, many significant achievements have been made in the manipulation of magnetostatic fields, ${ }^{[10-15]}$ thermal conduction, ${ }^{[16-19]}$ and electrostatic fields. ${ }^{[20-24]}$ In 2007, Wood and Pendry proposed a DC metamaterial that pointed the way towards the design of static magnetic cloaks, ${ }^{[10]}$ this DC metamaterial was experimentally verified soon afterwards. ${ }^{[11]}$ Recently, the DC magnetic cloak has been theoretically investigated $^{[12]}$ and experimentally realized using superconductors and ferromagnetic materials. ${ }^{[13,14]}$ By using the same materials as DC magnetic cloaks, the theoretical realization of a DC magnetic concentrator is also demonstrated. ${ }^{[15]}$ On the basis of form invariance of the heat conduction equation, transformation thermodynamics has been investigated to manipulate diffusive heat flow; ${ }^{[16]}$ through tailoring inhomogeneity and anisotropy of conductivities, transient thermal cloaking has been experimentally demonstrated. ${ }^{[17]}$ In addition, manipulation of heat flux with only two kinds of materials (by utilizing a multilayered composite approach) has been reported. ${ }^{[18,19]}$ Recently, a transformation-optics-based DC electric cloak, composed of inhomogeneous and anisotropic conductivities, has been implemented using an anisotropic and spatially-varying network of resistors. ${ }^{[20]}$ Soon after, an ultrathin DC electric cloak ${ }^{[21]}$ and a DC electric concentrator ${ }^{[22]}$ were reported using similar resistor networks. More recently, an exterior DC cloak ${ }^{[23]}$ and an active DC cloak ${ }^{[24]}$ have been experimentally realized by the use of inhomogeneous and anisotropic conductivities with the aid of active sources. It is noted that EM invisibility cloaks made of $L-C$ networks have been experimentally demonstrated. ${ }^{[25]}$

Here, we demonstrate the designs of novel devices (viz., a cloaking sensor, bilayer cloak, and fan-shaped concentrator) for manipulating DC currents with natural bulk materials, and we further experimentally realize the bilayer cloak and fan-shaped concentrator to confirm the proposed methodology. The significance of this work is twofold. First, only the most common bulk materials are employed to construct the proposed devices; this does not involve exotic materials that need to be mimicked with complicated resistor networks, ${ }^{[20-24]}$ thus pushing the transformation devices a big step further towards practical applications. Second, our design schemes - derived rigorously from electric conduction equations - are exact rather than approximate ones. Furthermore, the proposed devices can be arbitrarily scaled up and down without changing the materials.

We begin with the concept of cloaking sensor in DC currents (which will naturally lead to the design of a bilayer cloak). A cloaking sensor ${ }^{[26]}$ is a sensor wrapped by a shell that is capable 

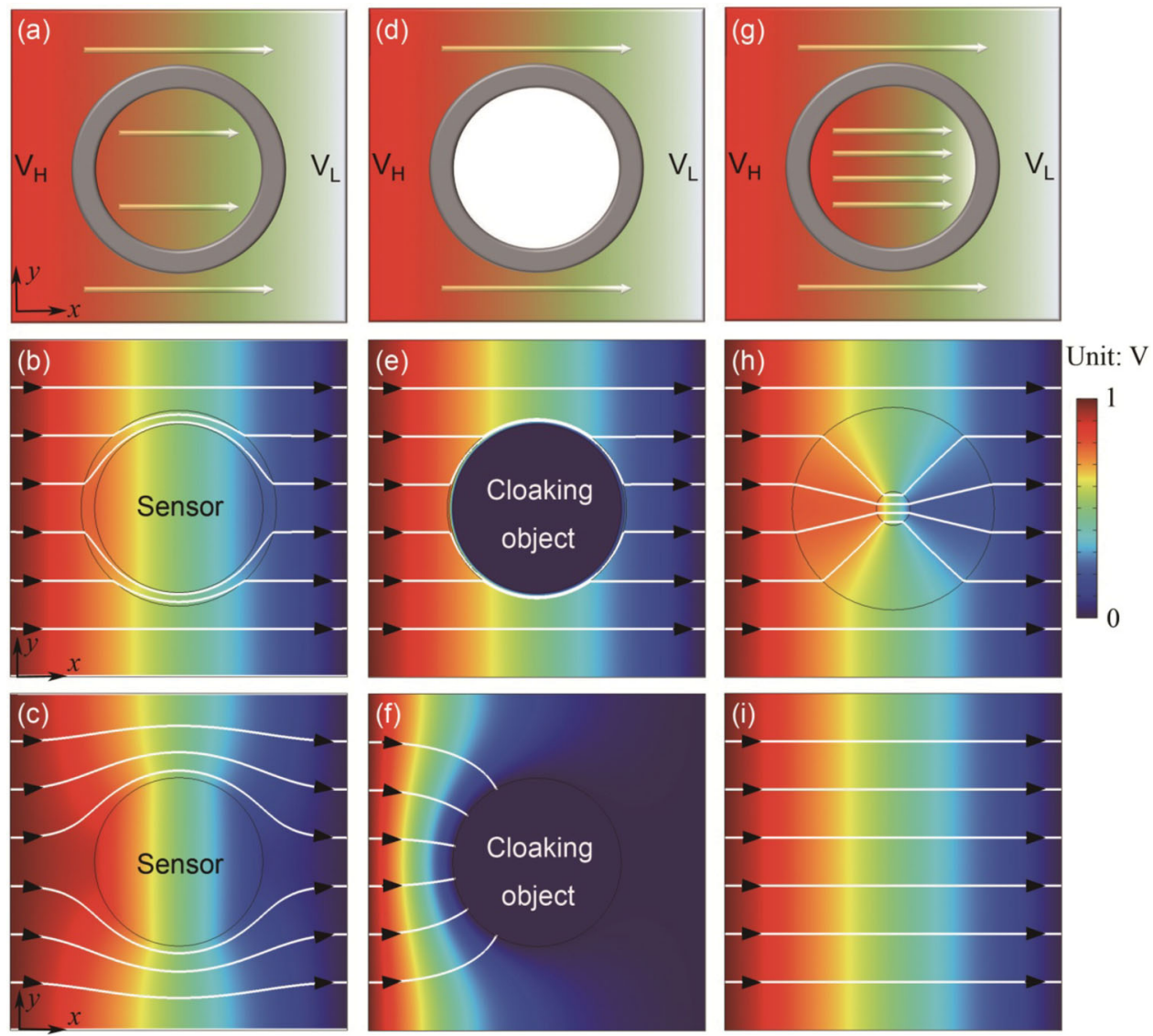

Figure 1. Demonstration of novel bilayer DC devices for manipulation of DC currents: a) cloaking sensor; b) simulation results for cloaking sensor; c) simulation results for bare sensor; d) DC bilayer cloak; e) simulation results for the bilayer cloak; f) simulation results for a bare and grounded object; g) DC electric concentrator; h) simulation results for the concentrator; and, i) simulation results for a pure background without the concentrator. DC currents are represented with arrow lines.

of receiving an incoming signal without distorting the external field. Figure 1a demonstrates the concept of a cloaking sensor in DC currents. We consider a round sensor (with radius $b$ ) wrapped by a shell (with thickness $c-b$ ). The conductivities of the sensor and shell are $\sigma_{1}$ and $\sigma_{2}$, respectively. A uniform DC current conducts in the $x$-direction with current density of $J_{0}$, which is equivalent to a uniform external electric field $E_{0}$ applied in the $x$-direction due to $\vec{J}=\sigma \vec{E}$. Since the electric potentials satisfy Laplace's equation $\nabla^{2} \phi=0$ in all regions of space, they can be generally expressed as

$\phi_{i}=\sum_{m=1}^{\infty}\left[A_{m}^{i} r^{m}+B_{m}^{i} r^{-m}\right] \cos m \theta$

where $A_{m}^{i}$ and $B_{m}^{i}(i=1,2,3)$ are constants to be determined by the boundary conditions and $\phi_{i}$ denotes the potential in different regions: $i=1$ for the cloaking region (where $r \leq b$ ), $i=$ 2 for the cloaking shell (where $b<r \leq c$ ), and $i=3$ for the exterior region (where $r>c$ ).

Taking into account that $\phi_{3}$ should tend to $-E_{0} r \cos \theta$ when $r \rightarrow \infty$, we only need to consider $m=1$. Since $\phi_{1}$ is limited when $r \rightarrow 0$, we can infer that $B_{1}^{1}=0$. As the electric potential and the normal component of electric field vector are continuous across the interfaces, we have $\left\{\left.\phi_{i}\right|_{r=b, c}=\left.\left.\phi_{i+1}\right|_{r=b, c} \sigma_{i} \frac{\partial \phi_{i}}{\partial r}\right|_{r=b, c}=\left.\sigma_{i+1} \frac{\partial \phi_{i+1}}{\partial r}\right|_{r=b, c}\right.$

Here, $\sigma_{3}=\sigma_{b}$, where $\sigma_{b}$ is the electric conductivity of the background. By substituting Equation 1 into Equation 2, we obtain

$B_{1}^{3}=E_{0} c^{2} \frac{\sigma_{2}\left(Q_{1}-Q_{2}\right)-\sigma_{b}\left(Q_{1}+Q_{2}\right)}{\sigma_{2}\left(Q_{1}-Q_{2}\right)+\sigma_{b}\left(Q_{1}+Q_{2}\right)}$

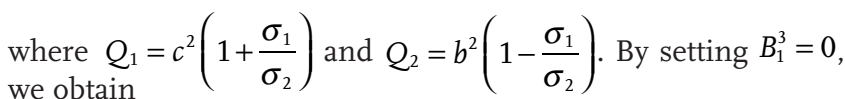

$c=b \sqrt{\frac{\left(\sigma_{2}-\sigma_{1}\right)\left(\sigma_{2}+\sigma_{b}\right)}{\left(\sigma_{2}+\sigma_{1}\right)\left(\sigma_{2}-\sigma_{b}\right)}}$

Obviously, a cloaking sensor can be successfully achieved as long as Equation 4 is satisfied. Considering that the sensor (central region), cloaking shell, and background are stainless steel (with $\sigma_{1}=1.3 \times 10^{6} \mathrm{~S} \mathrm{~m}^{-1}$ ), copper (with $\sigma_{2}=5.9 \times 10^{7}$ $\mathrm{S} \mathrm{m}^{-1}$ ), and iron (with $\sigma_{3}=1 \times 10^{7} \mathrm{~S} \mathrm{~m}^{-1}$ ), respectively, we obtain $c=2.9 \mathrm{~cm}$ when $b=2.5 \mathrm{~cm}$ according to Equation 4 . The simulated plot reproduced in Figure $1 \mathrm{~b}$ affirms the concept of a 
cloaking sensor in DC currents compared to the case of a bare sensor in Figure 1c.

The drawback of a cloaking sensor is that the cloaking shell has to be changed when either the geometrical size or material of the sensor is changed. Actually, in most cases, we only need to render an object invisible without receiving the incoming signal. Analogous to EM cloaks that prohibit incident waves with a PEC (perfect electric conductor) layer, an insulating layer $(a<r<b)$ located between the object and cloaking shell may prevent the electric current from touching the object. We thus derive a bilayer cloak, as conceptually demonstrated in Figure $1 \mathrm{~d}$. By setting $\sigma_{1}=0$, we obtain

$c=b \sqrt{\frac{\sigma_{2}+\sigma_{b}}{\sigma_{2}-\sigma_{b}}}$

Hence, an exact bilayer cloak, derived directly from the electric conduction equation, has been obtained. Equation 5 implies that the geometrical size of the bilayer cloak ( $b$ and $c$ ) can be tuned at will without changing the materials of the outer layer and background (i.e., $\sigma_{2}$ and $\sigma_{b}$ are fixed).

The experimental realization of a bilayer cloak is schematically illustrated in Figure 2a, which is composed of an insulating layer (where $a<r<b$ ) and a copper shell (where $b<r<c$ ).
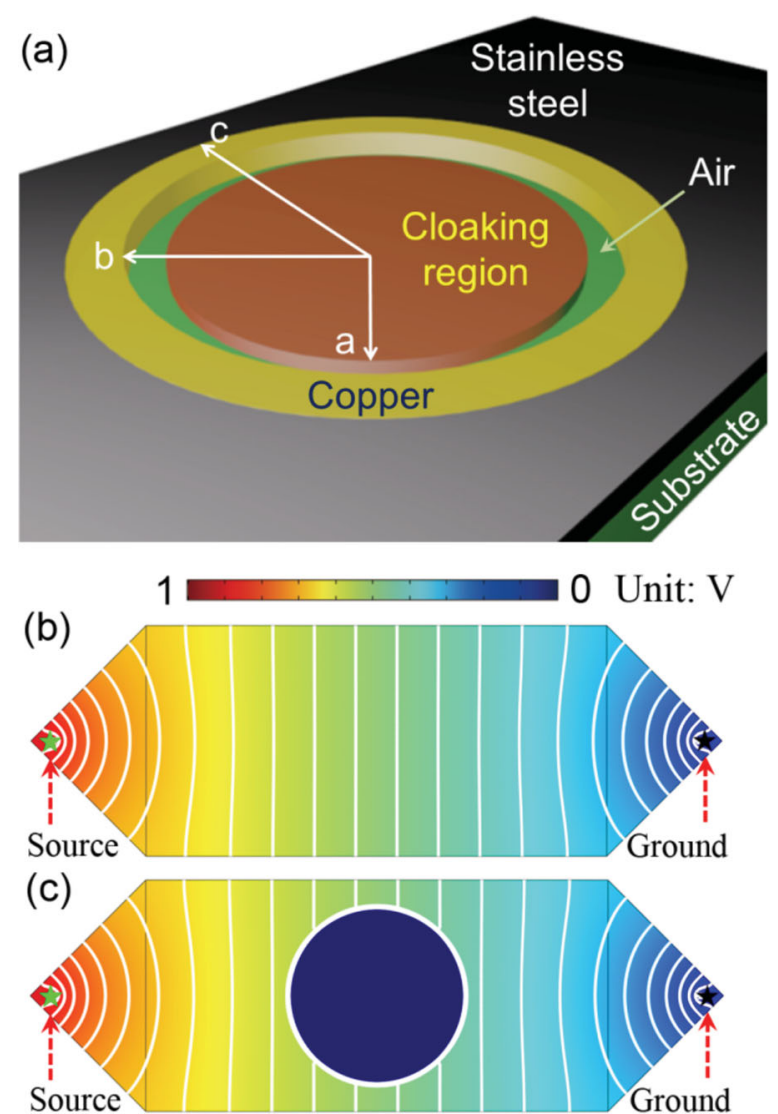

Figure 2. a) Schematic illustration for experimental realization of a DC bilayer cloak. b) Potential distribution of a homogeneous gradually changing structure. c) Potential distribution for the practical bilayer cloak in experiment. Equipotential lines are also demonstrated with white color in panel.
Here, air is wisely chosen as the insulating layer, which can be very thin due to its good insulating property. The geometrical parameters are $a=2.5 \mathrm{~cm}, b=2.6 \mathrm{~cm}$, and $c=2.66 \mathrm{~cm}$. We consider the case where the central region (cloaking region) is connected to the ground. The calculated potential distribution of bilayer cloak is plotted in Figure 1e, in which the DC currents (electric-field lines) are also presented. As expected, the DC currents bend conformally around the cloaking region and restore exactly outside the cloak without distortion, thus rendering the object invisible. When the bilayer cloak is removed, the simulation result of the bare object is demonstrated in Figure 1f, in which severe distortions of potential distribution and DC current lines can be clearly observed.

In the experimental setup, a gradually changing structure was adopted to transform circular equipotential lines to planar ones in the observation area. Figure $2 \mathrm{~b}$ shows the simulated potential distribution of a homogeneous gradually changing
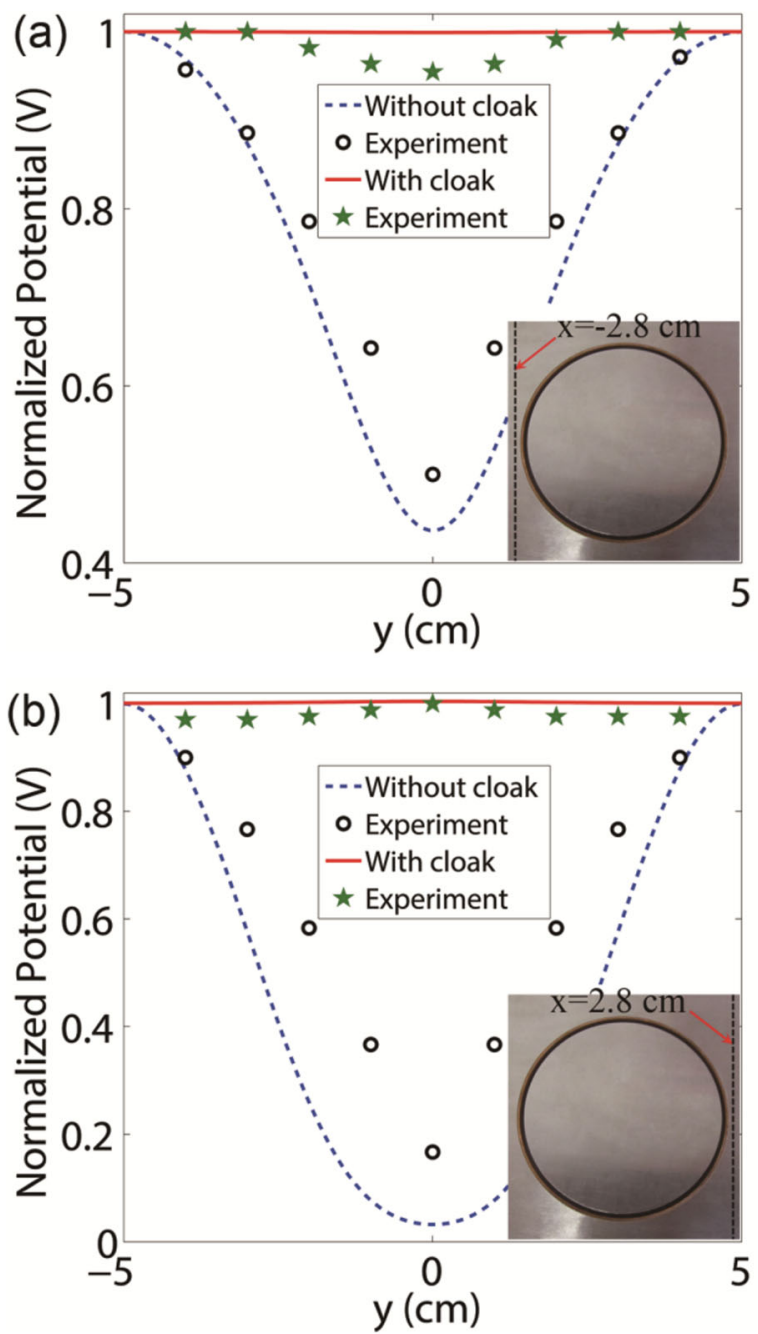

Figure 3. Simulation and experimental results of a bilayer cloak with $a$ $=2.5 \mathrm{~cm}, b=2.6 \mathrm{~cm}$, and $c=2.66 \mathrm{~cm}$. a) Normalized potential distribution at the left observation line $x=-2.8 \mathrm{~cm}$ presenting backward scattering. b) Normalized potential distribution at the right observation line $x=2.8 \mathrm{~cm}$ presenting forward scattering. Insets denote the position of observation lines. 


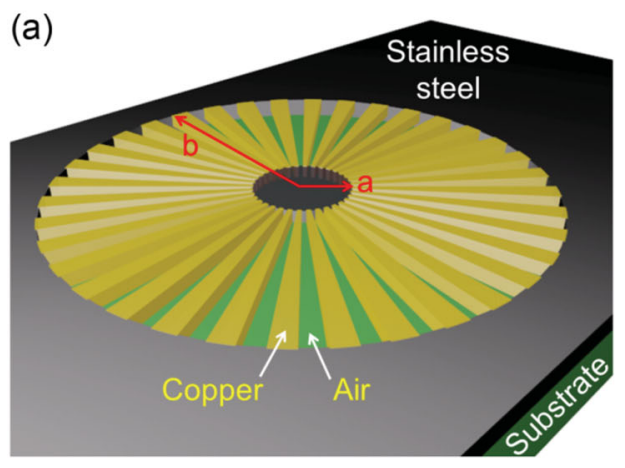

(c)

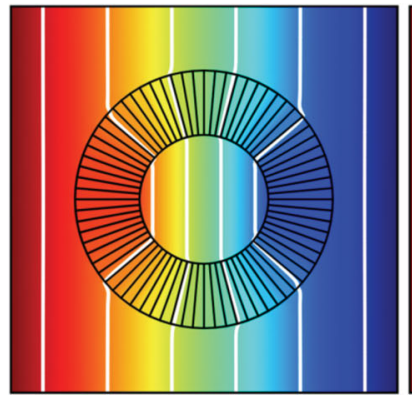

(d)

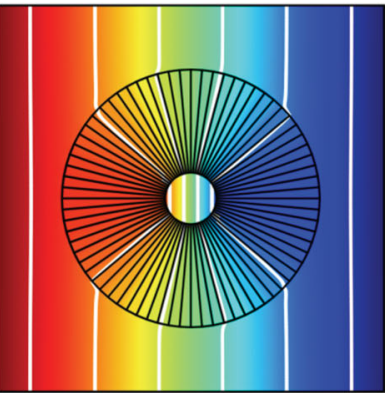

(b)

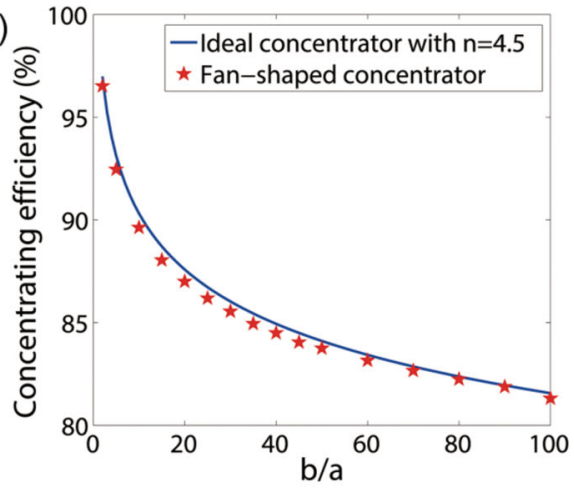

(e)

Unit: V

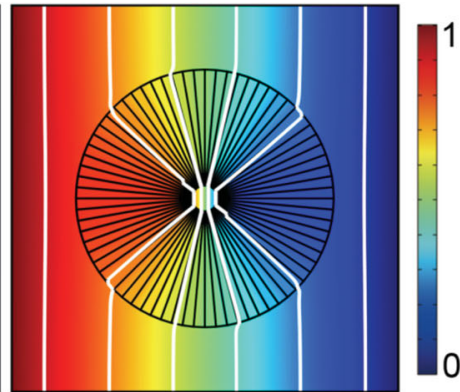

Figure 4. a) Schematic illustration for experimental realization of fan-shaped concentrator. b) Calculated concentrating efficiency for ideal concentrator with $n=4.5$ and fan-shaped concentrator in (a). Simulated potential distribution with different $b / a: c) b / a=2, \mathrm{~d}) b / a=5$, and, e) $b / a=10$. Equipotential lines are also indicated in white.

structure: it is clear that planar equipotential lines have been successfully obtained in observation area. The inner layer, outer layer, and background of the fabricated bilayer cloak are air, copper, and stainless steel, respectively. Its geometrical parameters are chosen to be the same as before: $a=2.5 \mathrm{~cm}, b=$ $2.6 \mathrm{~cm}$, and $c=2.66 \mathrm{~cm}$. The simulation result demonstrated in Figure 2c agrees very well with the pure background in Figure 2b. In the experimental setup, a DC power supply with 1 $\mathrm{V}$ magnitude is used as the source, and the voltage is measured using a FLUKE 45 Dual Display Multimeter.

The cloaking performance can be evaluated by measuring the potential distribution along the observation lines (shown as black dotted lines in the insets of Figure 3) near the bilayer cloak. In the ideal case, the observation lines are straight equipotential lines. The simulated and measured results are shown in Figure 3, in which (a) and (b) correspond to the normalized potential distribution at the left observation line (where $x=-2.8 \mathrm{~cm}$ ) and the right observation line (where $x=2.8 \mathrm{~cm}$ ), respectively. As expected, without the cloak, the presence of an object strongly distorts the original equipotential lines. When the central region is wrapped by the bilayer cloak, both forward or backward scattering are eliminated and the potential profiles restore exactly to the original equipotential lines (represented by straight lines). It is clear that the measurement agrees well with simulation, which validates our design scheme.

To further demonstrate that our proposed scheme is robust, we design and fabricate a bilayer cloak with a background of iron (see the Supporting Information). An exact cloak has to satisfy two conditions: i) the external field should be repelled from the cloaked region, and, ii) the external field outside the cloak should be undisturbed (as if nothing is there). Our bilayer cloak completely fulfills this ideal case based on the existence of an exact solution from the conduction equation.

Conceptually demonstrated in Figure 1g, a DC electric concentrator can enhance the electric field and current density in a given region without distorting the external field. The concentrator can be divided into three regions: the focusing region (where $r<a$ ), shell region (where $a \leq r \leq b$ ), and external region (where $r>b$ ). Both the focusing region and external region have the same electric conductivity $\sigma_{b}$. We assume that the electric conductivity of the shell region is homogeneous but anisotropic with $\sigma_{r}=2^{n} \sigma_{b}$ and $\sigma_{\theta}=2^{-n} \sigma_{b}$, where $n>0$. Considering that a uniform electric field $E_{0}$ is externally applied in the $x$-direction, the potential for the three regions can be obtained:

$$
\begin{aligned}
\phi_{1} & =-E_{0}\left(\frac{b}{a}\right)^{1-l} r \cos \theta, \phi_{2} \\
& =-E_{0}\left(\frac{b}{r}\right)^{1-l} r \cos \theta, \phi_{3}=-E_{0} r \cos \theta
\end{aligned}
$$

where $l=\sqrt{\sigma_{\theta} / \sigma_{r}}=2^{-n}$. Clearly, we obtain $\phi_{1}=\phi_{2}=\phi_{3}$ if $n=0$, which represents free space. If $n \rightarrow \infty$, we obtain $\phi_{1} / \phi_{3}=b / a$, which means that $100 \%$ concentrating efficiency is achieved. Figure $1(\mathrm{~h})$ shows the simulated potential distribution with the same geometrical parameters as those in the concentrating experiment: $a=1.2 \mathrm{~cm}$ and $b=6 \mathrm{~cm}$. Obviously, the DC current is focused into the central region without distortion. As a 
reference, the simulation result of a pure background is also demonstrated in Figure 1i.

On the basis of effective media theory (EMT), homogeneous but anisotropic material may be practically realized by alternatively stacking two natural materials in the azimuthal direction; the experimental realization of such a fan-shaped concentrator is shown in Figure 4a where copper wedges are periodically placed with air gaps. To quantitatively examine the concentrating efficiency of our proposed concentrator, we calculate $C E=|\phi|_{x=a}-\left.\left.\phi\right|_{x=-a}|/| \phi\right|_{x=b}-\left.\phi\right|_{x=-b} \mid$. Interestingly, we note from Figure $4(\mathrm{~b})$ that the concentrating efficiency of a fan-shaped concentrator is comparable with an ideal anisotropic concentrator with $n=4.5$; here the concentrating efficiency of the ideal anisotropic concentrator is defined as $C E=(b / a)^{-l}$. Clearly, the concentrating efficiency of the fan-shaped concentrator is still above $80 \%$ even when $b / a=100$. Furthermore, the fan-shaped concentrator (employing only copper) always keeps the external potential distribution undisturbed no matter what the ratio $b / a$ is, as shown in Figures 4(c-e). We observe that, outside the concentrator, the potential distribution is not distorted, which is the same as those in the homogeneous materials. According to $\vec{J}=\sigma \nabla \phi$, we obtain the current density of the central region as $\vec{J}_{\text {center }}=\vec{J}_{\text {backround }} \cdot C E \cdot b / a$, where $\vec{J}_{\text {background }}$ denotes the current density of the background. It is calculated that the current density of the concentrating region will be enhanced by more than 80 times when $b / a=100$.

We fabricated the fan-shaped concentrator in Figure 4(a) with $a=1.2 \mathrm{~cm}$ and $b=6 \mathrm{~cm}$, including 36 copper wedges and 36 air wedges. Analogous to the bilayer cloak, a gradually changing structure is also adopted to transform circular equipotential lines to planar ones in the observation area. An exact concentrator has to satisfy two conditions: i) the external field outside the concentrator should be undisturbed, and, ii) the electric field or current density should be focused into a smaller region. To examine the first condition, we measured and simulated the normalized potentials along the observation lines at $x=-7$ and $7 \mathrm{~cm}$, corresponding to the dotted black lines in Figure 5a, respectively. It is apparent that the potential profiles are the same as if there were nothing there. To examine the second condition, we measured and simulated the potential distribution along the observation line at $y=0$, as shown in Figure 5b. Compared to the pure background with linear potential distribution, the concentrator makes the voltage change sharply in the central region, which unambiguously demonstrates the concentrating effect. The measurement results agree well with simulation results, which confirm that our fan-shaped concentrator completely fulfills these two conditions.

In summary, we have demonstrated the manipulation of $D C$ currents with natural bulk materials, and experimentally confirm the methodology through before bilayer cloak and fanshaped concentrator. Our design schemes do not rely on transformation optics, and we can thus avoid the problems present in previous proposals (such as inhomogeneous and anisotropic parameters that need to mimicked via complicated resistor networks $\left.{ }^{[20-24]}\right)$. Also, the proposed schemes, derived directly from change to: electrical conduction equations, are exact rather than approximate ones. Finally, excellent performance can be
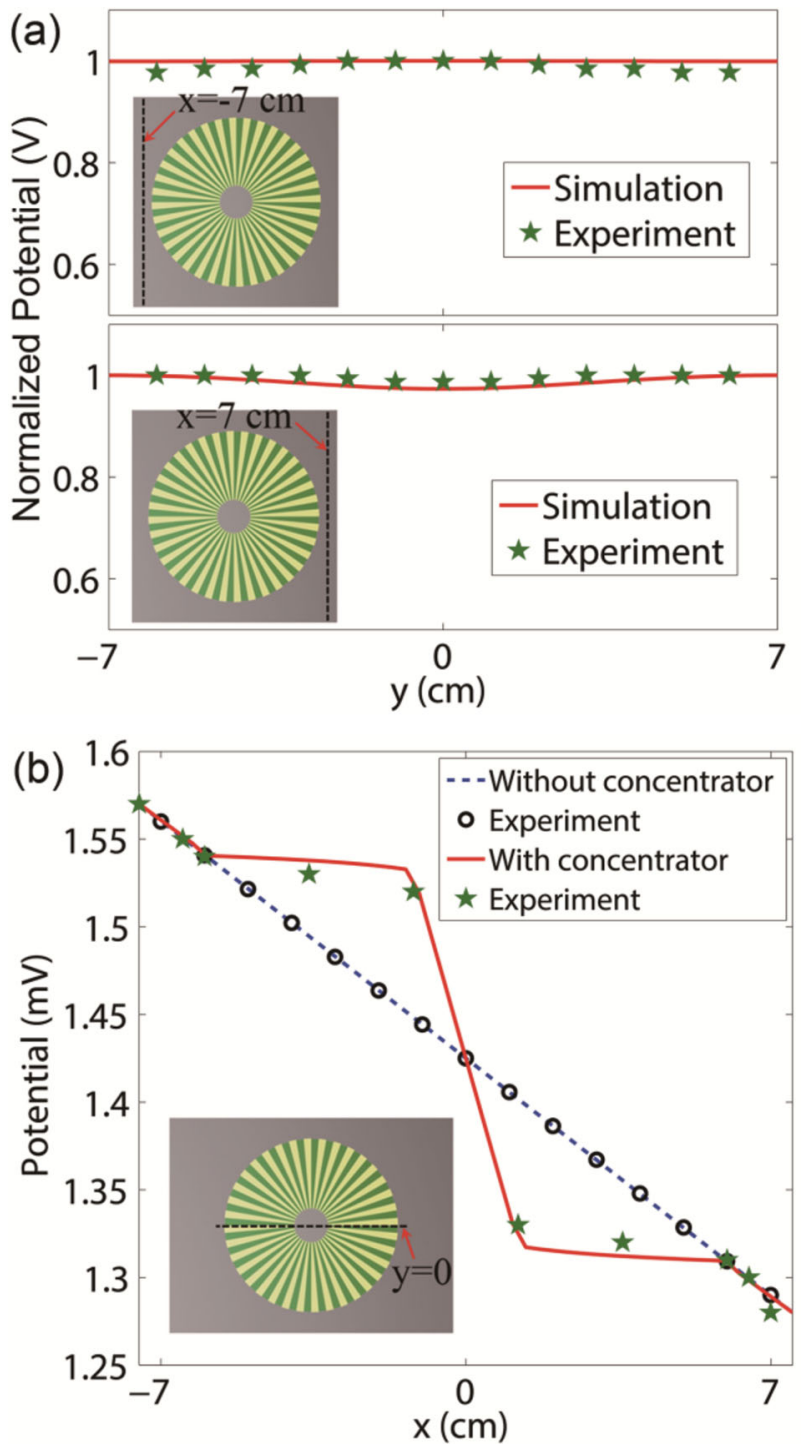

Figure 5. Simulation and measurement results of fan-shaped concentrator with $a=1.2 \mathrm{~cm}$ and $b=6 \mathrm{~cm}$. a) Normalized potential distribution at the observation lines $x=-7$ and $7 \mathrm{~cm}$, presenting backward scattering and forward scattering, respectively. b) The simulated and measured potential distributions along a line $y=0$. Insets denote the position of observation lines.

achieved by employing only natural bulk materials, thus indicating that our advanced scheme may be readily extended for various applications beyond DC control. ${ }^{[7,13,14,18,27,28]}$

\section{Supporting Information}

Supporting Information is available from the Wiley Online Library or from the author.

\section{Acknowledgements}

T.H., H.Y., and Y.L. contributed equally to this paper. S.Z. acknowledges the support from a Marie Curie fellowship under the career integration 
scheme. T.H. acknowledges the support of the National Science Foundation of China under grant no. 11304253. CWQ acknowledges MINDEF-NUS JPP grant R-263-000-A38-232 and R-263-000-A38-133 from the National University of Singapore.

Received: November 11, 2013 Revised: December 14, 2013 Published online: March 18, 2014

[1] A. Alù, N. Engheta, Phys. Rev. E 2005, 72, 016623.

[2] A. Alù, N. Engheta, Phys. Rev. Lett. 2008, 100, 113901.

[3] J. B. Pendry, D. Schurig, D. R. Smith, Science 2006, 312, 1780.

[4] U. Leonhardt, Science 2006, 312, 1777.

[5] M. Rahm, D. Schurig, D. A. Roberts, S. A. Cummer, D. R. Smith, J. B. Pendry, Photon. Nanostruct. Fundam. Appl. 2008, 6, 87.

[6] A. D. Yaghjian, S. Maci, New J. Phys. 2008, 10, 115200.

[7] S. Zhang, C. Xia, N. Fang, Phys. Rev. Lett. 2011, 106, 024301.

[8] S. Zhang, D. A. Genov, C. Sun, X. Zhang, Phys. Rev. Lett. 2008, 100, 123002.

[9] M. Brun, S. Guenneau, A. B. Movchan, Appl. Phys. Lett. 2009, 94, 061903.

[10] B. Wood, J. B. Pendry, J. Phys. Condens. Matter 2007, 19, 076208.

[11] F. Magnus, B. Wood, J. Moore, K. Morrison, G. Perkings, J. Fyson, M. C. K. Wiltshire, D. Caplin, L. F. Cohen, J. B. Pendry, Nat. Mater. 2008, 7, 295.
[12] A. Sanchez, C. Navau, J. Prat-Camps, D. X. Chen, New J. Phys. 2011, 13, 093034

[13] F. Gomory, M. Solovyov, J. Souc, C. Navau, J. Prat-Camps, A. Sanchez, Science 2012, 335, 1466.

[14] S. Narayana, Y. Sato, Adv. Mater. 2012, 24, 71.

[15] C. Navau, J. Prat-Camps, A. Sanchez, Phys. Rev. Lett. 2012, 109, 263903.

[16] S. Guenneau, C. Amra, D. Veynante, Opt. Express 2012, 20, 8207.

[17] R. Schittny, M. Kadic, S. Guenneau, M. Wegener, Phys. Rev. Lett. 2013, 110, 195901.

[18] S. Narayana, Y. Sato, Phys. Rev. Lett. 2012, 108, 214303.

[19] T. C. Han, T. Yuan, B. W. Li, C. W. Qiu, Sci. Rep. 2013, 3, 1593.

[20] F. Yang, Z. L. Mei, T. Y. Jin, T. J. Cui, Phys. Rev. Lett. 2012, 109, 053902.

[21] W. X. Jiang, C. Y. Luo, Z. L. Mei, T. J. Cui, Appl. Phys. Lett. 2013, 102, 014102.

[22] W. X. Jiang, C. Y. Luo, H. F. Ma, Z. L. Mei, T. J. Cui, Sci. Rep. 2012 2, 956.

[23] F. Yang, Z. L. Mei, X. Y. Yang, T. T. Jin, T. J. Cui, Adv. Funct. Mater. 2013, 23, 4306

[24] Q. Ma, Z. L. Mei, S. K. Zhu, T. Y. Jin, T. J. Cui, Phys. Rev. Lett. 2013, $111,173901$.

[25] C. Li, X. Liu, F. Li, Phys. Rev. B 2010, 81, 115133.

[26] A. Alù, N. Engheta, Phys. Rev. Lett. 2009, 102, 233901.

[27] S. Zhang, D. A. Dentcho, C. Sun, X. Zhang, Phys. Rev. Lett. 2008, 100, 123002.

[28] D. A. Dentcho, S. Zhang, X. Zhang, Nat. Phys. 2009, 5, 687. 